Chinese Journal of Organic Chemistry

\title{
五氟乙基硫叶立德: 一类新的亲电五氟乙基化试剂
}

\author{
刘亚飞 ${ }^{a}$ 葛航铭 ${ }^{a}$ 吕龙*,b沈其龙*, \\ $\left({ }^{a}\right.$ 中国科学院上海有机化学研究所 中国科学院有机氟化学重点实验室 分子合成科学卓越研究中心 \\ 中国科学院大学 上海 200032) \\ $\left({ }^{b}\right.$ 中国科学院上海有机化学研究所 上海 200032)
}

\begin{abstract}
摘要 发展了两个基于硫叶立德骨架的亲电五氟乙基化试剂, 其合成高效简洁、固体状态下稳定而溶液中反应活性高. 在温和条件下，该试剂可以与 $\beta$-酮酸酯、芳基/杂芳基碘化物以及富电子芳烃反应高产率地得到相应的五氟乙基化产物. 关键词 氟; 五氟乙基; 硫叶立德; 亲电
\end{abstract}

\section{Pentafluoroethyl-Substituted Sulfonium Ylides: New Electrophilic Pentafluoroethylating Reagents}

\author{
Liu, Yafei ${ }^{a} \quad$ Ge, Hangming ${ }^{a} \quad$ Lu, Long ${ }^{*, b} \quad$ Shen, Qilong*,a \\ ( ${ }^{a}$ Key Laboratory of Organofluorine Chemistry, Center for Excellence in Molecular Synthesis, Shanghai Institute of Organic \\ Chemistry, University of Chinese Academy of Sciences, Chinese Academy of Sciences, Shanghai 200032) \\ ( ${ }^{b}$ Shanghai Institute of Organic Chemistry, Chinese Academy of Sciences, Shanghai 200032)
}

\begin{abstract}
Two electrophilic pentafluoroethylating reagents of pentafluoroethyl(p-methylphenyl)sulfonium bis(carbomethoxy)methylide (4a) and pentafluoroethyl(4-nitrophenyl)sulfonium bis(carbomethoxy)methylide (4b), and their reactions under mild conditions with $\beta$-ketoesters, aryl iodides and heteroaromatics are described.

Keywords fluorine; pentafluoroethyl; sulfonium ylide; electrophilic
\end{abstract}

\section{Introduction}

In 2015, we discovered that trifluoromethyl-substituted sulfonium ylide, a shelf-stable crystalline solid, was an electrophilic trifluoromethylating reagent that was able to trifluoromethylate $\beta$-ketoesters in $\mathrm{N}, \mathrm{N}$-dimethylformamide (DMF) using $\mathrm{K}_{2} \mathrm{CO}_{3}$ as the base or aryl iodides in the presence of copper power (Figure 1). ${ }^{[1]}$ Classically, alkyl-substituted sulfonium ylides are generally acting as nucleophilic reagents for the preparation of epoxides, aziridine or cyclopropane derivatives, ${ }^{[2]}$ the unusual umpolung reactivity of the trifluoromethyl-substituted sulfonium ylide promoted to study difluoromethyl- or monofluoromethyl-substituted sulfonium ylide and found that both of them were highly electrophilic difluoromethylating/monofluoromethylating reagents. ${ }^{[3,4]}$ More specifically, in the presence of Lewis acid of $\mathrm{LiBF}_{4}$, primary and secondary alkanols reacted efficiently with reagent $\mathbf{2}$ to give<smiles>COC(=O)C(=O)[SH](c1ccccc1)C(F)(F)F</smiles>

1

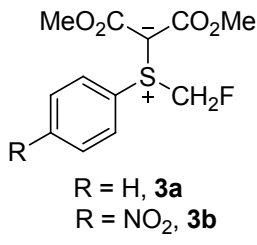<smiles>[R]O[N+](=O)c1ccc([S+](c2ccc([R])cc2)C(C(C)=O)C(C)=O)cc1</smiles><smiles>[R]c1ccc([Sb](C(C)=O)C(=O)C(C)=O)cc1</smiles>

Figure 1 Electrophilic fluoroalkylating reagents based on sulfonium ylide skeleton

\footnotetext{
*Corresponding authors. E-mail: lulong@mail.sioc.ac.cn; shenq1@mail.sioc.ac.cn

Received July 9, 2018; revised August 2, 2018; published online September 10, 2018.

Dedicated to Professor Qingyun Chen on the occasion of his 90th birthday.

Project supported by the National Natural Science Foundation of China (Nos. 21625206, 21632009, 21572258, 21572259, 21421002) and the Strategic Priority Research Program of the Chinese Academy of Sciences (No. XDB20000000).

国家自然科学基金(Nos. 21625206, 21632009, 21572258, 21572259, 21421002)、中国科学院战略性先导科技专项 B 类(No. XDB20000000)资助项目.
} 
difluoromethyl ethers in high yields, while reagent 3 reacted with a variety of nucleophiles such as alkoxides, aryl thiolates, amides and malonates to afford monofluoromethylated compounds in high yields.

In light of the high electrophilicity of the fluoromethylated sulfonium ylides, we wondered whether the sulfonium ylide skeleton is capable of acting as a general platform for the development of electrophilic reagents of other fluoroalkyl groups. We now disclose herein that two pentafluoroethyl-substituted sulfonium ylides $\mathbf{4 a}$ and $\mathbf{4 b}$ are highly electrophilic pentafluoroethylating reagents, which can pentafluoroethylate $\beta$-ketoesters, aryl iodides and heteroaromatics in high yields. Previously reported electrophilic pentafluoroethylating reagents including Yagupolskii and Umemoto's pentafluoroethyliodonium salts, ${ }^{[5]}$ Togni-type pentafluoroethylbenziodoxole $\left(\mathrm{BIXC}_{2} \mathrm{~F}_{5}\right)$ developed by Qing, Shen and their coworkers, ${ }^{[6]}$ and Yagupolskii-type pentafluoroethylated sulfonium salt developed by Xiao and co-workers. ${ }^{[7]}$ Thus, the development of sulfonium ylide-based pentafluoroethylating reagents $\mathbf{4 a}$ and $\mathbf{4 b}$ represents a complement to the powerful arsenal of electrophilic reagents for pentafluoroethylation (Figure 2).

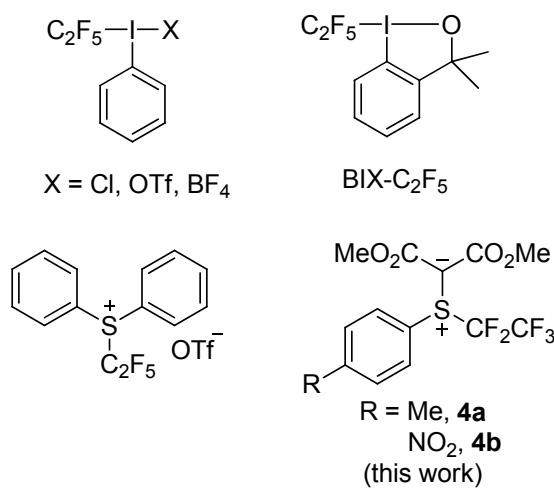

Figure 2 Previously reported electrophilic pentafluoroethylating reagents

\section{Results and discussion}

Pentafluoroethylating reagent of pentafluoroethyl( $p$ methyl phenyl)sulfonium bis(carbomethoxy)methylide (4a) was prepared in $61 \%$ yield on a $4.8 \mathrm{~g}$ scale via a one-pot two-step process. Treatment of 4-methylbenzenethiol with $\mathrm{C}_{2} \mathrm{~F}_{5} \mathrm{I}$ generated pentafluoroethyl 4-methylphenyl thioether. ${ }^{[8]}$ The crude product without further purification was allowed to react with dimethyl diazomalonate in the presence of $\mathrm{Rh}_{2}(\text { esp })_{2}\left(0.1 \mathrm{~mol} \%\right.$; esp $=\alpha, \alpha, \alpha^{\prime}, \alpha^{\prime}-$ tetramethyl-1,3-benzenedipropionic acid) in dichloromethane after $2 \mathrm{~h}$ at $40{ }^{\circ} \mathrm{C}$ to afford compound $4 \mathrm{a}$ in $61 \%$ yield. $^{[1]}$ Likewise, pentafluoroethyl(4-nitrophenyl)sulfonium bis(carbomethoxy)methylide (4b) was synthesized by the same procedure in $23 \%$ yield on a $6.8 \mathrm{~g}$ scale (Figure $3)$. Both compounds $\mathbf{4 a} \sim \mathbf{4 b}$ are air- and moisture-stable, crystalline white solids. No decomposition was observed after storing on bench for one-month.

To probe whether pentafluoroethyl-substituted sul-<smiles>[R]OC(=O)C(=N)C(=O)OC</smiles>

Figure 3 Preparation of pentafluoroethyl-substituted sulfonium ylides $\mathbf{4 a}$ and $\mathbf{4 b}$

fonium ylides $\mathbf{4 a / 4 b}$ can act as electrophilic pentafluoroethylating reagents, we first investigatd the reaction of $\beta$-ketoesters with compound $\mathbf{4 a} / \mathbf{4 b}$ in the presence of a variety of different bases and solvents. After a quick screening of the reaction conditions, it was found that the highest yield for the reaction of $\beta$-ketoester with $4 \mathbf{a}$ in DMSO was $64 \%$ when DBU was used as the base. The yield was greatly increased to $93 \%$ when compound $\mathbf{4 b}$ was used as the electrophilic pentafluoroethylating reagent. As shown in Table 1, a variety of five- or six-membered cyclic $\beta$-ketoesters can be pentafluoroethylated in good yields under mild conditions. Notably, the steric hinderance of the ester group does not have a significant effect on the yield of the reaction. Ester derived from methanol, ethanol, isopropanol or adamanol gave the desired corresponding pentafluoroethylated compounds in $82 \% \sim 98 \%$ yields $(\mathbf{5 c} \sim \mathbf{5 f})$.

Table 1 Scope for pentafluoroethylation of $\beta$-ketoesters with reagent $\mathbf{4} \mathbf{b}^{a}$
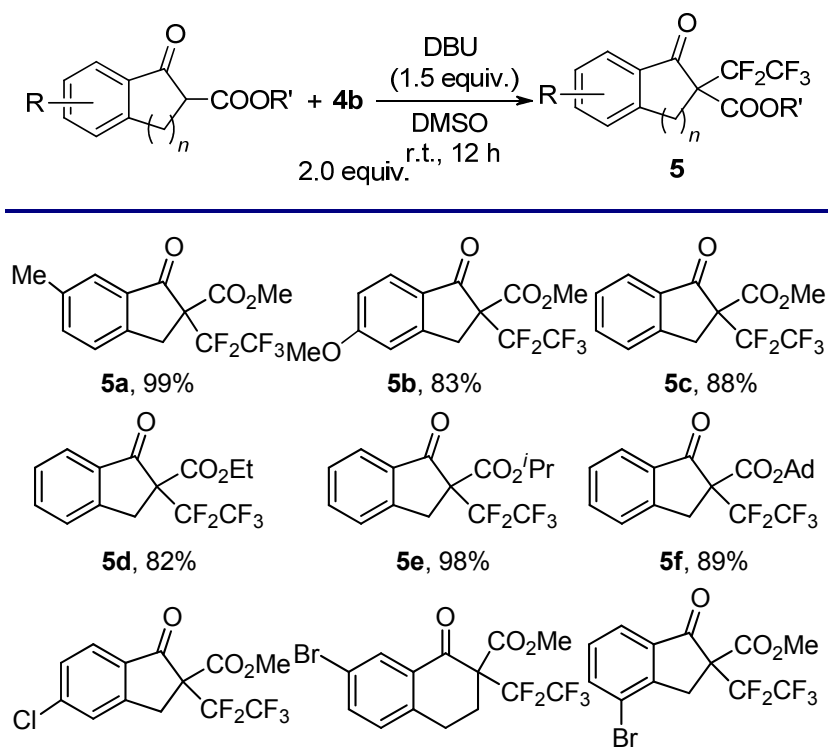

$5 g, 88 \%$

5h, $84 \%$

$5 i, 74 \%$

\begin{abstract}
${ }^{a}$ Reaction conditions: $\beta$-ketoester $(0.5 \mathrm{mmol}), 4 \mathbf{b}(1.0 \mathrm{mmol})$, DBU $(0.75 \mathrm{mmol})$ in DMSO $(3.0 \mathrm{~mL})$ at room temperature for 12 $\mathrm{h}$; Isolated yields.
\end{abstract}

Encouraged by the excellent reactivity of sulfonium ylide $4 \mathbf{b}$ with $\beta$-ketoesters, we next studied copper-mediated pentafluoroethylation of aryl iodides, as inspired by Xiao's copper-mediated reductive trifluoromethylation of 
aryl iodides with trifluoromethyl-substituted sulfonium salt. ${ }^{[9]}$ For this reaction, reagent $4 \mathbf{a}$ was more effective than reagent $\mathbf{4 b}$. It was discovered that solvent played an important role on the conversion of the pentafluoroethylation reaction. Specifically, reactions in DMF, $N$-methyl pyrrolidone (NMP) and dimethyl sulfoxide (DMSO) occurred in full conversion, while reactions in other solvents such as acetonitrile, dichloromethane, tetrahydrofuran (THF), or toluene occurred in much lower conversions. The reaction was sensitive to the reaction temperature. The highest yield was obtained when the reaction was conducted at $60{ }^{\circ} \mathrm{C}$. Common functional groups such as ester (6c), enolizable ketone (6b), nitro (6d), cyano (6e) and halogens including chloride, bromide (6f) were compatible. In addition, heteroaryl iodides including iodo pyridine $(\mathbf{6 h})$, pyrimidine (6i), benzothiophene (6k) or carbazole (6l) were all pentafluoroethylated in excellent yields (Table 2).

Table 2 Pentafluoroethylation of aryl iodides with reagent $\mathbf{4} \mathbf{a}^{a}$

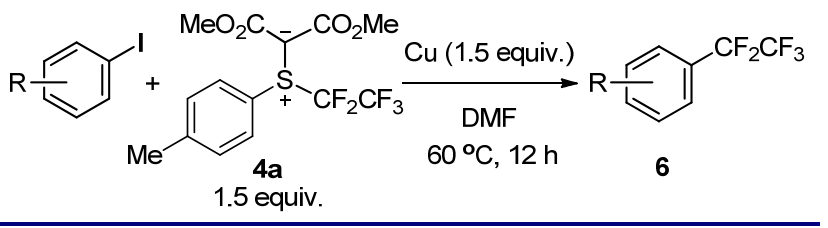<smiles>[R]c1ccc(C(F)(F)F)cc1</smiles>

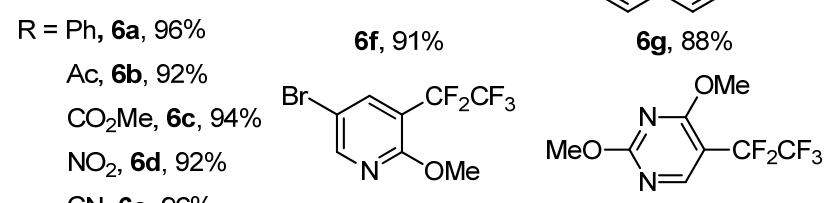
$\mathrm{CN}, \mathbf{6 e}, 96 \%$ $6 h, 89 \%$ 6i, $95 \%$
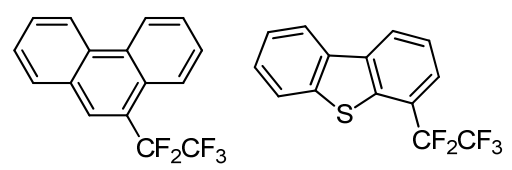<smiles>FC(F)(F)C(F)(F)C(F)(F)C(F)(F)C(F)(F)C(F)(F)F</smiles>

$6 \mathbf{j}, 88 \%$

$6 k, 98 \%$

6I, $99 \%$

${ }^{a}$ Reaction conditions: aryl iodide $(0.5 \mathrm{mmol}), 4 a(0.75 \mathrm{mmol})$, $\mathrm{Cu}(0.75 \mathrm{mmol})$ in DMF $(3.0 \mathrm{~mL})$ at $60{ }^{\circ} \mathrm{C}$ for $12 \mathrm{~h}$; Isolated yields.

Having successfully developed two pentafluoroethylating reactions with reagent $\mathbf{4 a} / \mathbf{4 b}$, we next tried to direct pentafluoroethylate the arene $\mathrm{C}-\mathrm{H}$ bonds, with anticipation that a pentafluoroethyl radical could be generated upon irradication by blue LED light. ${ }^{[10]}$ After a quick screening of the reaction conditions, the reaction worked smoothly to give pentafluoroethylated 1-phenylpyrrole in $70 \%$ yield, when the reaction was conducted in DMSO using 1,8-diazabicyclo[5.4.0]undec-7-ene (DBU) as the base. Interestingly, under the same reaction condition conditions, the formation of compound $7 \mathbf{a}$ was not observed when reagent 4a was used. Control experiment showed that the reaction did not occur in the absence of blue LED and the yield of the product decreased to $20 \%$ in the absence of DBU. In addition, the yield of the reaction de- creased significantly to $6 \%$ when 1.0 equivalent of radical inhibitor 2,2,6,6-tetramethyl-1-piperidinyloxy (TEMPO) was added, which suggests a radical pathway. Based on these results, a mechanism for this reaction was proposed. A donor-acceptor between reagent $\mathbf{4 b}$ and DBU is initially generated. Upon irradiation with blue LED, a pentafluoroethyl radical is formed, which reacts with arene to give pentafluoroethylated compounds. Because the electrophilic nature of the pentafluoroethyl group, only the electron-rich arenes such as indole and pyrrole with different substitution groups can be converted to pentafluoroethylated products in moderate to good yields (Table 3 ).

Table 3 Pentafluoroethylation of electron-rich heteroarenes with reagent $\mathbf{4} \mathbf{b}^{a}$

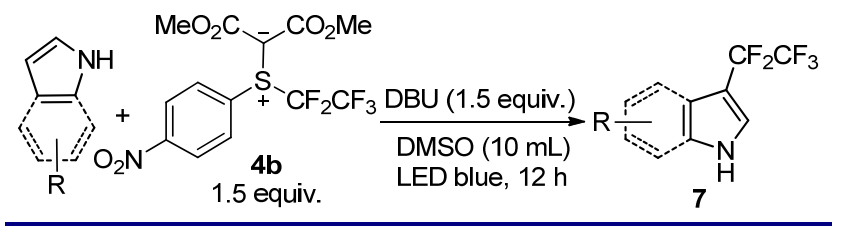

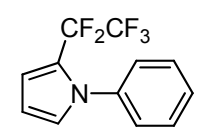

$7 a, 70 \%$

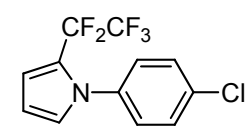

7b, $68 \%$

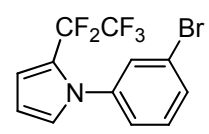

7c, $72 \%$<smiles>FC(F)(F)c1ccc(-n2cccc2C(F)(F)C(F)(F)F)cc1</smiles><smiles>Fc1ccc(-n2cccc2C(F)(F)C(F)(F)F)cc1</smiles>

7d, $68 \%$ $7 e, 66 \%$ 7f, $56 \%$<smiles>Fc1ccc(-c2[nH]c3ccccc3c2C(F)(F)C(F)(F)F)cc1Cl</smiles>

$7 g, 60 \%$<smiles>FC(F)(F)C(F)(F)C(F)(F)C(F)(F)C(F)(F)C(F)(F)F</smiles>

7h, $67 \%$

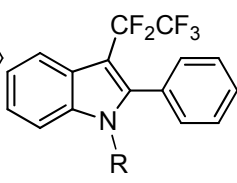

$\mathrm{R}=\mathrm{H}, \mathbf{7 i}, 61 \%$ Me, 7 j, $57 \%$
${ }^{a}$ Reaction conditions: heteroarene $(0.5 \mathrm{mmol}), 4 \mathbf{b}(0.75 \mathrm{mmol})$,
DBU $(0.75 \mathrm{mmol})$ in $10 \mathrm{~mL}$ DMSO under LED blue for $12 \mathrm{~h}$;
Isolated yields.

\section{Conclusions}

In summary, we have successfully invented two electrophilic pentafluoroethylating reagents $\mathbf{4 a} / \mathbf{4 b}$ from readily available starting materials. Importantly, reagents $\mathbf{4 a} / \mathbf{4 b}$ powerful electrophilic pentafluoroethylating reagents are able to pentafluoroethylate $\beta$-ketoesters, aryl iodides and heteroarenes to give the corresponding pentafluoroethylated products in high yields under mild conditions. Further expansion of the applications of these reagents is undergoing in our laboratory.

\section{Experimental section}

\subsection{General information}

All solvents were purified by standard method. ${ }^{1} \mathrm{H}$ NMR spectra were recorded on a $500 \mathrm{MHz}, 400 \mathrm{MHz}$ or 300 MHz. ${ }^{19}$ F NMR were recorded on a $376 \mathrm{MHz}$ or $282 \mathrm{MHz}$ 
spectrometer. ${ }^{1} \mathrm{H}$ NMR and ${ }^{13} \mathrm{C}$ NMR chemical shifts were determined relative to internal standard TMS at $\delta 0.0$ and ${ }^{19} \mathrm{~F}$ NMR chemical shifts were determined relative to $\mathrm{CFCl}_{3}$ as inter standard. Flash column chromatograph was carried out using 300 400 mesh silica gel at medium pressure.

Alkyl trifluoromethylthioethers were prepared according to procedure reported by Boiko. ${ }^{[8]}$ All other reagents were received from commercial sources. Solvents were freshly dried and degassed according to the purification handbook Purification of Laboratory Chemicals before using.

4.2 General procedure for the synthesis of pentafluoroethyl-substituted sulfonium ylide (4a)

4-Methylphenyl pentafluoroethyl thioether (4.84 g, 20.0 $\mathrm{mmol}), \mathrm{Rh}_{2}$ (esp) 2 (15 mg, $0.10 \mathrm{~mol} \%$ ) and $\mathrm{CH}_{2} \mathrm{Cl}_{2}(60 \mathrm{~mL})$ were placed into an oven-dried Schlenk tube that was equipped with a stirring bar under $\mathrm{N}_{2}$. Dimethyl diazomalonate (3.2 g, $20 \mathrm{mmol}$ ) was added dropwise. The tube was quickly sealed with a rubber stopper. The mixture was stirred at $40{ }^{\circ} \mathrm{C}$ for $2 \mathrm{~h}$. The mixture was then cooled to room temperature, and concentrated in vacuo. The residue was purified by flash chromatography [Eluent: $V$ (ethyl acetate $) / V$ (petroleum ether $)=1 / 3, R_{\mathrm{f}}=0.3$ ] to give pentafluoroethyl-(4-methylphenyl) bis(carbomethoxy) methylide (4a) as a white solid (4.5 g, 61\%). White solid, m.p. $32 \sim 34{ }^{\circ} \mathrm{C} ;{ }^{1} \mathrm{H}$ NMR (400 MHz, $\left.\mathrm{CDCl}_{3}, 293 \mathrm{~K}\right) \delta: 7.81(\mathrm{~d}$, $J=8.1 \mathrm{~Hz}, 2 \mathrm{H}), 7.34$ (d, $J=8.3 \mathrm{~Hz}, 2 \mathrm{H}), 3.75(\mathrm{~s}, 6 \mathrm{H}), 2.43$ $(\mathrm{s}, 3 \mathrm{H}) ;{ }^{19} \mathrm{~F}$ NMR $\left(375 \mathrm{MHz}, \mathrm{CDCl}_{3}\right) \delta$ : $-81.2(\mathrm{~s}, 3 \mathrm{~F}),-$ 97.9 (d, $J=206.6 \mathrm{~Hz}, 1 \mathrm{~F}),-101.4$ (d, $J=206.6 \mathrm{~Hz}, 1 \mathrm{~F})$; ${ }^{13} \mathrm{C}$ NMR (101 MHz, $\left.\mathrm{CDCl}_{3}, 293 \mathrm{~K}\right) \delta: 165.9,144.8$, $131.0,130.8,122.7,117.8$ (qt, $J=288.1,34.0 \mathrm{~Hz}), 111.5 \sim$ 118.9 (m), 55.0, 51.5, 21.4; IR (KBr) $v_{\max }$ : 2993, 2953, 2844, 2251, 1735, 1701, 1670, 1594, 1491, 1436, 1403, 1331, 1229, 1189, 1127, 1089, 1015, 964, 922, 810, 772, 747, 734, 700, 635, 627, 592, 547, $517 \mathrm{~cm}^{-1}$; MS (ESI): $373\left(\mathrm{M}^{+}+\mathrm{H}\right)$. HRMS (ESI) calcd for $\mathrm{C}_{14} \mathrm{H}_{14} \mathrm{~F}_{5} \mathrm{O}_{4} \mathrm{~S}$ : $373.0527\left(\mathrm{M}^{+}+\mathrm{H}\right)$, found 373.0528.

4.3 General procedure for the synthesis of pentafluoroethyl-substituted sulfonium ylide (4b)

4-Nitrophenyl pentafluoroethyl thioether $(6.8 \mathrm{~g}, 25$ $\mathrm{mmol}), \mathrm{Rh}_{2}$ (esp) 2 (19 mg, $0.10 \mathrm{~mol} \%$ ) and $\mathrm{CH}_{2} \mathrm{Cl}_{2}(60 \mathrm{~mL})$ were placed to an oven-dried Schlenk tube that was equipped with a stirring bar under $\mathrm{N}_{2}$. Dimethyl diazomalonate $(5.9 \mathrm{~g}, 37 \mathrm{mmol})$ was added dropwise. The tube was quickly sealed with a rubber stopper. The mixture was stirred at $40{ }^{\circ} \mathrm{C}$ for $12 \mathrm{~h}$. The mixture was then cooled to room temperature, and concentrated in vacuo. The residue was purified by flash chromatography [Eluent: $V$ (ethyl acetate $) / V$ (petroleum ether $)=1 / 2, R_{\mathrm{f}}=0.5$ ] to give pentafluoroethyl-(4-nitrophenyl) bis(carbomethoxy) methylide (4b) as yellow liquid (2.3 g, 23\%). Yellow liquid. ${ }^{1} \mathrm{H} \mathrm{NMR}$ (400 MHz, $\left.\mathrm{CDCl}_{3}, 293 \mathrm{~K}\right) \delta: 8.39$ (d, $\left.J=8.4 \mathrm{~Hz}, 2 \mathrm{H}\right), 8.03$ $(\mathrm{d}, J=8.5 \mathrm{~Hz}, 2 \mathrm{H}), 3.74(\mathrm{~s}, 6 \mathrm{H}) ;{ }^{19} \mathrm{~F}$ NMR $(375 \mathrm{MHz}$, $\left.\mathrm{CDCl}_{3}\right) \delta:-81.0(\mathrm{~s}, 3 \mathrm{~F}),-96.3(\mathrm{~d}, J=202.2 \mathrm{~Hz}, 1 \mathrm{~F})$, $-100.0(\mathrm{dq}, J=202.4 \mathrm{~Hz}, 3.7 \mathrm{~Hz}, 1 \mathrm{~F}) ;{ }^{13} \mathrm{C}$ NMR $(101$ $\left.\mathrm{MHz} \mathrm{CDCl}_{3}, 293 \mathrm{~K}\right) \delta: 165.4,150.3,132.5,131.1,124.9$, $117.5(\mathrm{qt}, J=288.3,33.8 \mathrm{~Hz}), 119.6 \sim 111.3(\mathrm{~m}), 52.7$, 52.0; IR (KBr) $v_{\max }: 3110,3001,2956,2847,1739,1710$, $1670,1604,1580,1533,1477,1437,1400,1331,1228$, $1128,1091,1010,963,916,854,800,773,746,723,679$, 636, 625, 596, $544 \mathrm{~cm}^{-1}$; MS (EI) m/z: 246 (100), 284, 403 $\left(\mathrm{M}^{+}\right)$. HRMS (EI) calcd for $\mathrm{C}_{13} \mathrm{H}_{10} \mathrm{O}_{6} \mathrm{~F}_{5} \mathrm{NS}$ : 403.0149, found 403.0142.

4.4 General procedure for the pentafluoroethylation of $\beta$-ketoesters

$\beta$-Ketoester $(0.5 \mathrm{mmol}), \mathrm{DBU}(114 \mathrm{mg}, 0.750 \mathrm{mmol}$, 1.50 equiv.) and $\mathbf{4 b}$ (403 mg, $1.00 \mathrm{mmol}, 2.00$ equiv.) were placed into an oven-dried Schlenk tube that was equipped with a stirring bar under $\mathrm{N}_{2}$. The tube added $3.0 \mathrm{~mL}$ of freshly distilled DMSO and the tube quickly sealed with a rubber stopper. The mixture was stirred at room temperature for 12 h. $20 \mathrm{~mL}$ of water and $40 \mathrm{~mL}$ of ether were added to the mixture, the organic phase was separated and extracted with water $(10 \mathrm{~mL} \times 5)$, dried over anhydrous $\mathrm{Na}_{2} \mathrm{SO}_{4}$, and concentrated in vacuo. The residue was purified by flash chromatography on silica gel.

Methyl 6-methyl-1-oxo-2-(pentafluoroethyl)-2,3-dihydro- $1 H$-indene-2-carboxylate $(\mathbf{5 a}){ }^{[3]}$ Eluent: $V($ ethyl acetate $) / V$ (petroleum ether $)=1 / 10, R_{\mathrm{f}}=0.4$. Yellow liquid (164 mg, 99\%). ${ }^{1} \mathrm{H} \mathrm{NMR}\left(400 \mathrm{MHz}, \mathrm{CDCl}_{3}, 293 \mathrm{~K}\right) \delta$ : 7.59 (s, 1H), 7.49 (d, $J=7.7 \mathrm{~Hz}, 1 \mathrm{H}), 7.40$ (d, $J=7.8 \mathrm{~Hz}$, $1 \mathrm{H}), 3.83$ (d, $J=17.6 \mathrm{~Hz}, 1 \mathrm{H}), 3.76(\mathrm{~s}, 3 \mathrm{H}), 3.54$ (d, $J=$ $17.6 \mathrm{~Hz}, 1 \mathrm{H}), 2.39(\mathrm{~s}, 3 \mathrm{H}) ;{ }^{19} \mathrm{~F}$ NMR $\left(375 \mathrm{MHz}, \mathrm{CDCl}_{3}\right) \delta$ : $-79.2(\mathrm{~s}, 3 \mathrm{~F}),-114.8(\mathrm{~d}, J=278.6 \mathrm{~Hz}, 1 \mathrm{~F}),-116.1(\mathrm{~d}$, $J=278.6 \mathrm{~Hz}, 1 \mathrm{~F})$; IR (KBr) $v_{\max }: 2959,2929,2868,1762$, $1728,1620,1588,1497,1436,1384,1335,1213,1097$, $1075,1031,993,911,876,853,820,791,755,736,688$, 648, 619, $535 \mathrm{~cm}^{-1}$; MS (EI) $\mathrm{m} / z: 243$ (100), 263, 291, 322 $\left(\mathrm{M}^{+}\right)$. HRMS (EI) calcd for $\mathrm{C}_{14} \mathrm{H}_{11} \mathrm{~F}_{5} \mathrm{O}_{3}: 322.0628$, found 322.0622 .

Methyl 6-methoxy-1-oxo-2-(pentafluoroethyl)-2,3-dihydro- $1 H$-indene-2-carboxylate $(\mathbf{5 b}):^{[3]}$ Eluent: $V($ ethyl acetate $) / V($ petroleum ether $)=1 / 10, R_{\mathrm{f}}=0.3$. Yellow solid $(140$ mg, 83\%), m.p. 78 80 ${ }^{\circ} \mathrm{C} ;{ }^{1} \mathrm{H}$ NMR (400 $\mathrm{MHz} \mathrm{CDCl}_{3}$, $293 \mathrm{~K}) \delta: 7.45(\mathrm{~d}, J=8.3 \mathrm{~Hz}, 1 \mathrm{H}), 7.31(\mathrm{~d}, J=7.3 \mathrm{~Hz}, 1 \mathrm{H})$, $7.26(\mathrm{~s}, 1 \mathrm{H}), 3.88(\mathrm{~s}, 3 \mathrm{H}), 3.83(\mathrm{~s}, 3 \mathrm{H}), 3.89$ (d, $J=17.4$ $\mathrm{Hz}, 1 \mathrm{H}), 3.57$ (d, $J=17.4 \mathrm{~Hz}, 1 \mathrm{H}) ;{ }^{19} \mathrm{~F}$ NMR $(375 \mathrm{MHz}$, $\left.\mathrm{CDCl}_{3}\right) \delta:-79.2(\mathrm{~s}, 3 \mathrm{~F}),-114.7(\mathrm{~d}, J=278.6 \mathrm{~Hz}, 1 \mathrm{~F})$, $-115.9(\mathrm{~d}, J=278.7 \mathrm{~Hz}, 1 \mathrm{~F})$; IR $(\mathrm{KBr}) v_{\max }: 3072,3011$, 2973, 2942, 2834, 1738, 1716, 1614, 1586, 1498, 1452, $1439,1429,1349,1336,1278,1219,1189,1160,1136$, $1116,1090,1060,1045,1021,990,973,959,921,892$, $853,837,817,774,758,743,700,684,674,639,604,560$, $528 \mathrm{~cm}^{-1}$; MS (EI) $\mathrm{m} / \mathrm{z}: 259,279,338\left(100, \mathrm{M}^{+}\right)$. HRMS (EI) calcd for $\mathrm{C}_{14} \mathrm{H}_{11} \mathrm{~F}_{5} \mathrm{O}_{4} 338.0578$, found 338.0574.

Methyl 1-oxo-2-(pentafluoroethyl)-2,3-dihydro-1 $H$-indene-2-carboxylate (5c): ${ }^{[3]}$ Eluent: $V($ ethyl acetate $) /$ $V($ petroleum ether $)=1 / 10, R_{\mathrm{f}}=0.3$. Yellow liquid $(135$ mg, 88\%). ${ }^{1} \mathrm{H}$ NMR (400 MHz, $\left.\mathrm{CDCl}_{3}, 293 \mathrm{~K}\right) \delta: 7.81(\mathrm{~d}$, $J=7.6 \mathrm{~Hz}, 1 \mathrm{H}), 7.68$ (t, $J=7.4 \mathrm{~Hz}, 1 \mathrm{H}), 7.52$ (d, $J=7.5$ $\mathrm{Hz}, 1 \mathrm{H}), 7.44$ (t, $J=7.4 \mathrm{~Hz}, 1 \mathrm{H}), 3.90$ (d, $J=17.7 \mathrm{~Hz}, 1 \mathrm{H})$, $3.78(\mathrm{~s}, 3 \mathrm{H}), 3.60(\mathrm{~d}, J=17.6 \mathrm{~Hz}, 1 \mathrm{H}) ;{ }^{19} \mathrm{~F}$ NMR $(375$ 
$\left.\mathrm{MHz}, \mathrm{CDCl}_{3}\right) \delta:-79.2(\mathrm{~s}, 3 \mathrm{~F}),-114.7(\mathrm{~d}, J=278.8 \mathrm{~Hz}$ $1 \mathrm{~F}),-116.1(\mathrm{~d}, J=278.8 \mathrm{~Hz}, 1 \mathrm{~F})$; IR $(\mathrm{KBr}) v_{\max }: 3041$, 2960, 2849, 1735, 1608, 1593, 1523, 1480, 1466, 1436, 1338, 1212, 1098, 1074, 1030, 992, 977, 914, 892, 867, $820,794,770,753,689,637,611,560,532 \mathrm{~cm}^{-1}$; MS (EI) $m / z: 229$ (100), 249, 277, $308\left(\mathrm{M}^{+}\right)$. HRMS (EI) calcd for $\mathrm{C}_{13} \mathrm{H}_{9} \mathrm{~F}_{5} \mathrm{O}_{3} 308.0472$, found 308.0471.

Ethyl 1-oxo-2-(pentafluoroethyl)-2,3-dihydro- $1 H$-indene-2-carboxylate $(\mathbf{5 d}):^{[3]}$ Eluent: $V($ ethyl acetate $) / V$ (petroleum ether $)=1 / 10, R_{\mathrm{f}}=0.4$. Yellow liquid $(132 \mathrm{mg}, 82 \%)$. ${ }^{1} \mathrm{H}$ NMR $\left(400 \mathrm{MHz}, \mathrm{CDCl}_{3}, 293 \mathrm{~K}\right) \delta: 7.81(\mathrm{~d}, J=7.6 \mathrm{~Hz}$, $1 \mathrm{H}), 7.68(\mathrm{t}, J=7.4 \mathrm{~Hz}, 1 \mathrm{H}), 7.52(\mathrm{~d}, J=7.7 \mathrm{~Hz}, 1 \mathrm{H}), 7.44$ $(\mathrm{t}, J=7.3 \mathrm{~Hz}, 1 \mathrm{H}), 4.34 \sim 4.15(\mathrm{~m}, 2 \mathrm{H}), 3.90(\mathrm{~d}, J=17.6$ $\mathrm{Hz}, 1 \mathrm{H}), 3.58$ (d, $J=17.6 \mathrm{~Hz}, 1 \mathrm{H}), 1.25$ (t, $J=7.1 \mathrm{~Hz}, 3 \mathrm{H})$; ${ }^{19} \mathrm{~F}$ NMR $\left(375 \mathrm{MHz}, \mathrm{CDCl}_{3}\right) \delta$ : $-79.0(\mathrm{~s}, 3 \mathrm{~F}),-114.4$ (d, $J=278.7 \mathrm{~Hz}, 1 \mathrm{~F}),-116.0(\mathrm{~d}, J=278.7 \mathrm{~Hz}, 1 \mathrm{~F})$; IR $(\mathrm{KBr}) v_{\max }$ : 2987, 1732, 1608, 1593, 1479, 1466, 1446, 1369, 1332, 1212, 1097, 1074, 1029, 974, 911, 866, 768, 688, $636 \mathrm{~cm}^{-1}$; MS (EI) m/z: 229 (100), 249, 277, 322 (M $\left.{ }^{+}\right)$. HRMS (EI) calcd for $\mathrm{C}_{14} \mathrm{H}_{11} \mathrm{~F}_{5} \mathrm{O}_{3}: 322.0628$, found 322.0623 .

Isopropyl 1-oxo-2-(pentafluoroethyl)-2,3-dihydro-1 $H$ indene-2-carboxylate (5e): ${ }^{[3]}$ Eluent: $V($ ethyl acetate $) /$ $V($ petroleum ether $)=1 / 10, R_{\mathrm{f}}=0.6$. Yellow liquid $(165$ mg, 98\%). ${ }^{1} \mathrm{H}$ NMR (400 MHz, $\left.\mathrm{CDCl}_{3}, 293 \mathrm{~K}\right) \delta: 7.80$ (d, $J=7.7 \mathrm{~Hz}, 1 \mathrm{H}), 7.67(\mathrm{t}, J=7.1 \mathrm{~Hz}, 1 \mathrm{H}), 7.52$ (d, $J=7.6$ $\mathrm{Hz}, 1 \mathrm{H}), 7.43$ (t, $J=7.3 \mathrm{~Hz}, 1 \mathrm{H}), 5.12 \sim 4.99(\mathrm{~m}, 1 \mathrm{H}), 3.87$ $(\mathrm{d}, J=17.6 \mathrm{~Hz}, 1 \mathrm{H}), 3.57(\mathrm{~d}, J=17.6 \mathrm{~Hz}, 1 \mathrm{H}), 1.22(\mathrm{~d}, J=$ $4.1 \mathrm{~Hz}, 6 \mathrm{H}) ;{ }^{19} \mathrm{~F}$ NMR $\left(375 \mathrm{MHz}, \mathrm{CDCl}_{3}\right) \delta$ : $-78.9(\mathrm{~s}$, $3 \mathrm{~F}),-114.2(\mathrm{~d}, J=278.5 \mathrm{~Hz}, 1 \mathrm{~F}),-115.9(\mathrm{~d}, J=278.5$ $\mathrm{Hz}, 1 \mathrm{~F})$; IR (KBr) $v_{\max }$ : 2987, 2941, 1732, 1608, 1594, 1467, 1390, 1378, 1335, 1266, 1148, 1103, 1074, 1025, 975, 923, 897, 866, 836, 793, 768, 753, 684, 634, 533 $\mathrm{cm}^{-1}$; MS (EI) m/z: 248 (100), 277, 294, $336\left(\mathrm{M}^{+}\right)$. HRMS (EI) calcd for $\mathrm{C}_{15} \mathrm{H}_{13} \mathrm{~F}_{5} \mathrm{O}_{3}: 336.0785$, found 336.0781.

Adamantan-1-yl 1-oxo-2-(pentafluoroethyl)-2,3-dihydro-1H-indene-2-carboxylate (5f): Eluent: $V$ (ethyl acetate) $/ V$ (petroleum ether $)=1 / 10, R_{\mathrm{f}}=0.6$. Yellow liquid (190 mg, 89\%). ${ }^{1} \mathrm{H}$ NMR (400 MHz, $\left.\mathrm{CDCl}_{3}, 293 \mathrm{~K}\right) \delta: 7.81$ $(\mathrm{d}, J=7.6 \mathrm{~Hz}, 1 \mathrm{H}), 7.66$ (t, $J=7.3 \mathrm{~Hz}, 1 \mathrm{H}), 7.51(\mathrm{~d}, J=7.6$ $\mathrm{Hz}, 1 \mathrm{H}), 7.43$ (t, $J=7.5 \mathrm{~Hz}, 1 \mathrm{H}), 3.82(\mathrm{~d}, J=17.5 \mathrm{~Hz}, 1 \mathrm{H})$, $3.54(\mathrm{~d}, J=17.5 \mathrm{~Hz}, 1 \mathrm{H}), 2.14(\mathrm{~s}, 3 \mathrm{H}), 2.06(\mathrm{~s}, 6 \mathrm{H}), 1.62$ $(\mathrm{s}, 6 \mathrm{H}) ;{ }^{19} \mathrm{~F}$ NMR $\left(375 \mathrm{MHz}, \mathrm{CDCl}_{3}\right) \delta$ : $-78.6(\mathrm{~s}, 3 \mathrm{~F})$, $-113.7(\mathrm{~d}, J=278.3 \mathrm{~Hz}, 1 \mathrm{~F}),-115.8(\mathrm{~d}, J=278.2 \mathrm{~Hz}$, 1F); $\left.{ }^{13} \mathrm{C} \mathrm{NMR} \mathrm{(101} \mathrm{MHz,} \mathrm{CDCl}_{3}, 293 \mathrm{~K}\right) \delta: 192.8,162.6$ (d, $J=7.6 \mathrm{~Hz}), 151.6,136.0,134.2,128.2,126.1,125.3$, 84.7, 77.4, 77.0, 76.7, 63.4, 63.3, 63.2, 63.0, 40.8, 35.9, $33.6(\mathrm{~m}), 30.9$; IR (KBr) $v_{\max }: 2915,2855,1728,1608$, 1593, 1479, 1466, 1321, 1258, 1212, 1096, 1074, 7049, $1024,974,964,940,912,884,863,771,734,688,648 \mathrm{~cm}$ ${ }^{-1}$; MS (EI) $m / z$ : 135 (100), $428\left(\mathrm{M}^{+}\right)$. HRMS (EI) calcd for $\mathrm{C}_{22} \mathrm{H}_{21} \mathrm{~F}_{5} \mathrm{O}_{3}: 428.1411$, found 428.1409.

Methyl 5-chloro-1-oxo-2-(pentafluoroethyl)-2,3-dihydro-1H-indene-2-carboxylate $\mathbf{( 5 g )})^{[3]}$ Eluent: $V($ ethyl acetate $) / V$ (petroleum ether $)=1 / 10, R_{\mathrm{f}}=0.5$. Yellow solid $(150$ mg, 88\%), m.p. 50 52 ${ }^{\circ} \mathrm{C} ;{ }^{1} \mathrm{H}$ NMR (400 MHz, $\mathrm{CDCl}_{3}$, $293 \mathrm{~K}) \delta: 7.73(\mathrm{~d}, J=8.1 \mathrm{~Hz}, 1 \mathrm{H}), 7.52(\mathrm{~s}, 1 \mathrm{H}), 7.41$ (d,
$J=8.1 \mathrm{~Hz}, 1 \mathrm{H}), 3.88(\mathrm{~d}, J=17.8 \mathrm{~Hz}, 1 \mathrm{H}), 3.79(\mathrm{~s}, 3 \mathrm{H})$, $3.57(\mathrm{~d}, J=17.9 \mathrm{~Hz}, 1 \mathrm{H}) ;{ }^{19} \mathrm{~F}$ NMR $\left(375 \mathrm{MHz}, \mathrm{CDCl}_{3}\right) \delta$ : $-79.2(\mathrm{~s}, 3 \mathrm{~F}),-114.6(\mathrm{~d}, J=278.9 \mathrm{~Hz}, 1 \mathrm{~F}),-116.2(\mathrm{~d}$, $J=278.9 \mathrm{~Hz}, 1 \mathrm{~F})$; IR (KBr) $v_{\max }: 2960,1764,1735,1601$, $1586,1436,1323,1262,1209,1152,1100,1070,1029$, 992, 976, 892, 848, 826, 789, 735, 636, 613, $534 \mathrm{~cm}^{-1}$; MS (EI) $m / z: 263,283,311,342$ (100) $\left(\mathrm{M}^{+}\right), 344$ (34.7). HRMS (EI) calcd for $\mathrm{C}_{13} \mathrm{H}_{8} \mathrm{~F}_{5} \mathrm{O}_{3} \mathrm{Cl}$ : 342.0082, found 342.0075 .

Methyl 7-bromo-1-oxo-2-(pentafluoroethyl)-1,2,3,4-tetrahydronaphthalene-2-carboxylate $(\mathbf{5 h}):{ }^{[3]}$ Eluent: $V$ (ethyl acetate $) / V($ petroleum ether $)=1 / 10, R_{\mathrm{f}}=0.3$. White solid (162 mg, 84\%), m.p. $119 \sim 121{ }^{\circ} \mathrm{C} ;{ }^{1} \mathrm{H}$ NMR (400 MHz, $\left.\mathrm{CDCl}_{3}, 293 \mathrm{~K}\right) \delta: 8.19(\mathrm{~s}, 1 \mathrm{H}), 7.62(\mathrm{~d}, J=8.0 \mathrm{~Hz}, 1 \mathrm{H})$, $7.14(\mathrm{~d}, J=8.1 \mathrm{~Hz}, 1 \mathrm{H}), 3.75$ (s, 3H), 2.98 (s, 2H), 2.86 (d, $J=13.7 \mathrm{~Hz}, 1 \mathrm{H}), 2.54 \sim 2.39(\mathrm{~m}, 1 \mathrm{H}) ;{ }^{19} \mathrm{~F}$ NMR $(375$ $\left.\mathrm{MHz} \mathrm{CDCl}_{3}\right) \delta:-76.5(\mathrm{~s}, 3 \mathrm{~F}),-111.3(\mathrm{~d}, J=279.9 \mathrm{~Hz}$, $1 \mathrm{~F}),-112.8(\mathrm{~d}, J=280.0 \mathrm{~Hz}, 1 \mathrm{~F})$; IR $(\mathrm{KBr}) v_{\max }: 3078$, 3058, 2964, 1729, 1705, 1589, 1476, 1456, 1437, 1405, $1351,1310,1285,1271,1219,1171,1150,1132,1109$, 1080, 1065, 1014, 976, 905, 890, 859, 833, 746, 721, 657, 611, $541 \mathrm{~cm}^{-1}$; MS (EI) $\mathrm{m} / z: 281$ (100), 283, 400 (25.4), 402 (25.4). HRMS (EI) calcd for $\mathrm{C}_{14} \mathrm{H}_{10} \mathrm{~F}_{5} \mathrm{O}_{3} \mathrm{Br} 399.9733$, found 399.9732 .

Methyl 4-bromo-1-oxo-2-(Pentafluoroethyl)-2,3-dihydro-1H-indene-2-carboxylate (5i): Eluent: $V$ (ethyl acetate $/ V$ (petroleum ether $)=1 / 10, R_{\mathrm{f}}=0.47$. Yellow liquid (143 mg, 74\%); ${ }^{1} \mathrm{H}$ NMR (400 MHz, $\left.\mathrm{CDCl}_{3}, 293 \mathrm{~K}\right) \delta$ : 7.85 (d, $J=7.7 \mathrm{~Hz}, 1 \mathrm{H}), 7.77(\mathrm{~d}, J=7.5 \mathrm{~Hz}, 1 \mathrm{H}), 7.36(\mathrm{t}$, $J=7.7 \mathrm{~Hz}, 1 \mathrm{H}), 3.83(\mathrm{~d}, J=19.0 \mathrm{~Hz}, 1 \mathrm{H}), 3.80(\mathrm{~s}, 3 \mathrm{H})$, $3.50(\mathrm{~d}, J=18.2 \mathrm{~Hz}, 1 \mathrm{H}) ;{ }^{19} \mathrm{~F}$ NMR $\left(375 \mathrm{MHz}, \mathrm{CDCl}_{3}\right) \delta$ : $-79.0(\mathrm{~s}, 3 \mathrm{~F}),-114.4(\mathrm{~d}, J=279.0 \mathrm{~Hz}, 1 \mathrm{~F}),-115.9$ (d, $J=279.1 \mathrm{~Hz}, 1 \mathrm{~F}) ;{ }^{13} \mathrm{C} \mathrm{NMR}\left(101 \mathrm{MHz}, \mathrm{CDCl}_{3}, 293 \mathrm{~K}\right) \delta$ : $191.5,164.3$ (d, $J=7.1 \mathrm{~Hz}), 151.1,139.0,135.8,130.2$, $124.2,121.5,118.7$ (qt, $J=288.5,36.1 \mathrm{~Hz}$ ), $116.7 \sim 110.3$ (m), 62.3 (dd, $J=22.9,18.8 \mathrm{~Hz}), 54.01,34.4$ (d, $J=2.7$ $\mathrm{Hz}$ ); IR (KBr) $v_{\max }$ : 2959, 1766, 1735, 1598, 1459, 1437, 1332, 1254, 1216, 1173, 1128, 1101, 1077, 1028, 976, 908, 881, 809, 784, 755, 730, $644 \mathrm{~cm}^{-1}$; MS (EI) $\mathrm{m} / \mathrm{z}: 307$ (100), 327, 355, 386 (67.3), 388 (69.1). HRMS (EI) calcd for $\mathrm{C}_{13} \mathrm{H}_{8} \mathrm{~F}_{5} \mathrm{O}_{3} \mathrm{Br}$ 385.9577, found 385.9570.

4.5 General procedure for the pentafluoroethylation of aryl iodides

Aryl iodide (0.5 mmol), Cu powder (48 mg, $0.75 \mathrm{mmol}$, 1.5 equiv.) and $4 \mathbf{a}$ (279 mg, $0.750 \mathrm{mmol}, 1.50$ equiv.) were placed into an oven-dried Schlenk tube that was equipped with a stirring bar under $\mathrm{N}_{2} .3 .0 \mathrm{~mL}$ of freshly distilled DMF was added and the tube was quickly sealed with a rubber stopper. The mixture was stirred at $60{ }^{\circ} \mathrm{C}$ for $12 \mathrm{~h}$. $20 \mathrm{~mL}$ of water and $40 \mathrm{~mL}$ of ether were added to the mixture. The organic phase was separated and extracted with water $(10 \mathrm{~mL} \times 5)$, dried over anhydrous $\mathrm{Na}_{2} \mathrm{SO}_{4}$, and concentrated in vacuo. The residue was purified by flash chromatography on silica gel.

4-(Pentafluoroethyl)-1,1'-biphenyl (6a): ${ }^{[3]}$ Eluent: petroleum ether $\left(R_{\mathrm{f}}=0.6\right)$. White solid (130 mg, 96\%), m.p. 
$69 \sim 71{ }^{\circ} \mathrm{C} ;{ }^{1} \mathrm{H}$ NMR (400 MHz, $\left.\mathrm{CDCl}_{3}, 293 \mathrm{~K}\right) \delta: 7.81 \sim$ $7.68(\mathrm{~m}, 4 \mathrm{H}), 7.65$ (d, $J=7.2 \mathrm{~Hz}, 2 \mathrm{H}), 7.52$ (t, $J=7.4 \mathrm{~Hz}$, 2H), $7.49 \sim 7.41(\mathrm{~m}, 1 \mathrm{H}) ;{ }^{19} \mathrm{~F}$ NMR $\left(375 \mathrm{MHz}, \mathrm{CDCl}_{3}\right) \delta$ : $-84.8(\mathrm{~s}, 3 \mathrm{~F}),-114.7$ (s, 2F); IR (KBr) $v_{\max }: 3084,1612$, 1569, 1490, 1453, 1407, 1339, 1295, 1273, 1204, 1165, $1150,1131,1114,1092,1024,1006,975,954,917,840$, 768, 739, 724, 690, 655, $628 \mathrm{~cm}^{-1}$; MS (EI) m/z: 203 (100), 272. HRMS (ESI) calcd for $\mathrm{C}_{14} \mathrm{H}_{9} \mathrm{~F}_{5} 272.0624$, found 272.0616 .

1-(4-(Pentafluoroethyl)phenyl)ethanone $(\mathbf{6 b})^{[3]}$ Eluent: $V($ ethyl acetate $) / V($ petroleum ether $)=1 / 20, R_{\mathrm{f}}=0.3$. Yellow liquid (110 mg, 92\%); ${ }^{1} \mathrm{H}$ NMR (400 $\mathrm{MHz}, \mathrm{CDCl}_{3}$, $293 \mathrm{~K}) \delta: 8.06(\mathrm{~d}, J=8.0 \mathrm{~Hz}, 2 \mathrm{H}), 7.70(\mathrm{~d}, J=8.2 \mathrm{~Hz}, 2 \mathrm{H})$, $2.64(\mathrm{~s}, 3 \mathrm{H}) ;{ }^{19} \mathrm{~F}$ NMR $\left(375 \mathrm{MHz}, \mathrm{CDCl}_{3}\right) \delta$ : $-84.8(\mathrm{~s}$, $3 \mathrm{~F}),-115.6(\mathrm{~s}, 2 \mathrm{~F})$; IR $(\mathrm{KBr}) v_{\max }: 1697,1578,1509$, 1410, 1361, 1336, 1288, 1266, 1209, 1149, 1099, 1075, 1021, 976, 957, 912, 833, 744, 725, 647, 607, $590 \mathrm{~cm}^{-1}$; MS (EI) $m / z: 223$ (100), 238. HRMS (EI) calcd for $\mathrm{C}_{10} \mathrm{H}_{7} \mathrm{~F}_{5} \mathrm{O} 238.0417$, found 238.0420.

Methyl 4-(pentafluoroethyl)benzoate $(\mathbf{6 c}):^{[3]}$ Eluent: $V($ ethyl acetate $) / V($ petroleum ether $)=1 / 20, R_{\mathrm{f}}=0.4$. Yellow liquid (119 mg, 94\%); ${ }^{1} \mathrm{H}$ NMR (400 MHz, $\mathrm{CDCl}_{3}$, $293 \mathrm{~K}) \delta: 8.16(\mathrm{~d}, J=8.4 \mathrm{~Hz}, 2 \mathrm{H}), 7.68(\mathrm{~d}, J=8.3 \mathrm{~Hz}, 2 \mathrm{H})$, $3.96(\mathrm{~s}, 3 \mathrm{H}) ;{ }^{19} \mathrm{~F}$ NMR $\left(375 \mathrm{MHz}, \mathrm{CDCl}_{3}\right) \delta$ : $-84.7(\mathrm{~s}$, $3 \mathrm{~F}),-115.5(\mathrm{~s}, 2 \mathrm{~F})$; IR $(\mathrm{KBr}) v_{\max }: 3005,2958,2850$, $1736,1617,1582,1514,1439,1413,1338,1314,1285$, 1208, 1150, 1096, 1024, 976, 964, 860, 828, 773, 759, 712, 696, $639 \mathrm{~cm}^{-1}$; MS (EI) m/z: 223 (100), 254. HRMS (EI) calcd for $\mathrm{C}_{10} \mathrm{H}_{7} \mathrm{~F}_{5} \mathrm{O}_{2} 254.0366$, found 254.0364.

1-Nitro-4-(pentafluoroethyl)benzene $\quad(\mathbf{6 d}):^{[3]}$ Eluent: $V($ ethyl acetate $) / V($ petroleum ether $)=1 / 20, R_{\mathrm{f}}=0.5$. Yellow liquid (110 mg, 92\%); ${ }^{1} \mathrm{H}$ NMR (400 MHz, $\mathrm{CDCl}_{3}$, $293 \mathrm{~K}) \delta: 8.38(\mathrm{~d}, J=8.5 \mathrm{~Hz}, 2 \mathrm{H}), 7.83(\mathrm{~d}, J=8.5 \mathrm{~Hz}$, $2 \mathrm{H}) ;{ }^{19} \mathrm{~F}$ NMR $\left(282 \mathrm{MHz}, \mathrm{CDCl}_{3}\right) \delta$ : -84.9 (s, 3F), -115.9 (s, 2F); IR (KBr) $v_{\text {max }}: 3124,3092,2879,1617$, 1537, 1416, 1355, 1287, 1208, 1153, 1098, 1019, 977, 961, $911,853,760,738,710,692,636 \mathrm{~cm}^{-1}$; MS (EI) $\mathrm{m} / \mathrm{z}: 145$, 172, 203, 219, 241. HRMS (EI) calcd for $\mathrm{C}_{8} \mathrm{H}_{4} \mathrm{NF}_{5} \mathrm{O}_{2}$ : 241.0162, found 241.0157.

4-(Pentafluoroethyl)benzonitrile $(\mathbf{6 e}):^{[3]}$ Eluent: $V$ (ethyl acetate $) / V$ (petroleum ether $)=1 / 20, R_{\mathrm{f}}=0.7$. Yellow liquid (106 mg, 96\%). ${ }^{1} \mathrm{H}$ NMR (400 MHz, $\left.\mathrm{CDCl}_{3}, 293 \mathrm{~K}\right) \delta$ : $7.82(\mathrm{~d}, J=8.0 \mathrm{~Hz}, 2 \mathrm{H}), 7.74(\mathrm{~d}, J=8.2 \mathrm{~Hz}, 2 \mathrm{H}) ;{ }^{19} \mathrm{~F}$ NMR $\left(375 \mathrm{MHz}, \mathrm{CDCl}_{3}\right) \delta$ : $-89.0(\mathrm{~s}, 3 \mathrm{~F}),-120.2(\mathrm{~s}, 2 \mathrm{~F})$; IR $(\mathrm{KBr}) v_{\max }: 3108,3063,2237,1931,1616,1577,1508$, 1412, 1334, 1286, 1262, 1208, 1154, 1100, 1024, 976, 959, $914,840,746,730,645,601,554 \mathrm{~cm}^{-1}$; MS (EI) $\mathrm{m} / \mathrm{z}: 152$ (100), 221. HRMS (EI) calcd for $\mathrm{C}_{9} \mathrm{H}_{4} \mathrm{~F}_{5} \mathrm{~N} 221.0264$, found 221.0267 .

4-Bromo-2-chloro-1-(pentafluoroethyl)benzene (6f): Eluent: petroleum ether, $R_{\mathrm{f}}=0.9$; Colorless liquid (141 mg, 91\%). ${ }^{1} \mathrm{H}$ NMR (400 MHz, $\left.\mathrm{CDCl}_{3}, 293 \mathrm{~K}\right) \delta: 7.70$ (s, 1H), $7.54(\mathrm{~d}, J=9.3 \mathrm{~Hz}, 1 \mathrm{H}), 7.48(\mathrm{~d}, J=8.5 \mathrm{~Hz}, 1 \mathrm{H}) ;{ }^{19} \mathrm{~F}$ NMR $\left(375 \mathrm{MHz}, \mathrm{CDCl}_{3}\right) \delta:-87.3(\mathrm{~s}, 3 \mathrm{~F}),-115.1(\mathrm{~s}, 2 \mathrm{~F}) ;{ }^{13} \mathrm{C}$ NMR $\left(101 \mathrm{MHz}, \mathrm{CDCl}_{3}, 293 \mathrm{~K}\right) \delta: 134.8,134.3(\mathrm{t}, J=2.6$ $\mathrm{Hz}), 130.7$ (t, $J=8.3 \mathrm{~Hz}), 130.3,127.0,125.4(\mathrm{t}, J=46.5$ Hz), 119.0 (qt, $J=287.1,38.4 \mathrm{~Hz}$ ), $116.1 \sim 110.9(\mathrm{~m})$; IR
(KBr) $v_{\max }: 3100,2927,2855,1587,1557,1478,1377$, 1334, 1285, 1212, 1168, 1132, 1111, 1089, 1059, 969, 954, 874, 821, 792, 746, 667, $640 \mathrm{~cm}^{-1}$; MS (EI) $\mathrm{m} / \mathrm{z}: 241$ (100), 308 (21.24), 310 (28.34). HRMS (EI) calcd for $\mathrm{C}_{8} \mathrm{H}_{3} \mathrm{~F}_{5} \mathrm{BrCl}$ 307.9027, found 307.9023.

1-(Pentafluoroethyl)naphthalene $(\mathbf{6 g}):{ }^{[11]}$ Eluent: petroleum ether, $R_{\mathrm{f}}=0.8$. Colorless liquid $(108 \mathrm{mg}, 88 \%) ;{ }^{1} \mathrm{H}$ NMR (400 MHz, $\left.\mathrm{CDCl}_{3}, 293 \mathrm{~K}\right) \delta: 8.28(\mathrm{~d}, J=8.1 \mathrm{~Hz}$, $1 \mathrm{H}), 8.05(\mathrm{~d}, J=8.1 \mathrm{~Hz}, 1 \mathrm{H}), 7.93(\mathrm{~d}, J=7.6 \mathrm{~Hz}, 1 \mathrm{H}), 7.86$ $(\mathrm{d}, J=7.2 \mathrm{~Hz}, 1 \mathrm{H}), 7.73 \sim 7.40(\mathrm{~m}, 3 \mathrm{H}) ;{ }^{19} \mathrm{~F}$ NMR $(375$ $\left.\mathrm{MHz}, \mathrm{CDCl}_{3}\right) \delta$ : $-88.2(\mathrm{~s}, 3 \mathrm{~F}),-113.1(\mathrm{~s}, 2 \mathrm{~F})$.

5-Bromo-2-methoxy-3-(pentafluoroethyl)pyridine (6h): Eluent: petroleum ether, $R_{\mathrm{f}}=0.6$. Colorless liquid (136 mg, $89 \%) ;{ }^{1} \mathrm{H}$ NMR (400 MHz, $\left.\mathrm{CDCl}_{3}, 293 \mathrm{~K}\right) \delta: 8.38(\mathrm{~s}, 1 \mathrm{H})$, $7.91(\mathrm{~s}, 1 \mathrm{H}), 3.99(\mathrm{~s}, 3 \mathrm{H}) ;{ }^{19} \mathrm{~F}$ NMR $\left(375 \mathrm{MHz}, \mathrm{CDCl}_{3}\right) \delta$ : $-87.4(\mathrm{~s}, 3 \mathrm{~F}),-117.6(\mathrm{~s}, 2 \mathrm{~F})$; IR $(\mathrm{KBr}) v_{\max }: 2960,2931$, 2859, 1592, 1566, 1476, 1416, 1400, 1309, 1272, 1246, 1205, 1147, 1119, 1081, 1014, 985, 912, 846, 774, 757, $694 \mathrm{~cm}^{-1}$; MS (EI) m/z: 225 (100), 305 (2.72), 307 (2.28). HRMS (EI) calcd for $\mathrm{C}_{8} \mathrm{H}_{5} \mathrm{NF}_{5} \mathrm{OBr} 304.9475$, found 304.9474 .

2,4-Dimethoxy-5-(pentafluoroethyl)pyrimidine (6i): Eluent: $V($ ethyl acetate $) / V$ (petroleum ether $)=1 / 10, R_{\mathrm{f}}=0.5$. Colorless liquid (123 mg, 95\%); ${ }^{1} \mathrm{H}$ NMR (400 MHz, $\left.\mathrm{CDCl}_{3}, 293 \mathrm{~K}\right) \delta: 8.40(\mathrm{~s}, 1 \mathrm{H}), 4.03(\mathrm{~s}, 6 \mathrm{H}) ;{ }^{19} \mathrm{~F} \mathrm{NMR}(375$ $\left.\mathrm{MHz} \mathrm{CDCl}_{3}\right) \delta:-84.6(\mathrm{~s}, 3 \mathrm{~F}),-113.8(\mathrm{~s}, 2 \mathrm{~F})$; IR $(\mathrm{KBr})$ $v_{\max }: 3003,2963,1605,1564,1478,1406,1350,1302$, 1269, 1250, 1207, 1131, 1097, 1066, 1013, 977, 954, 805, 754, 698, 680, 657, $586 \mathrm{~cm}^{-1}$; MS (EI) m/z: 258 (100). HRMS (EI) calcd for $\mathrm{C}_{8} \mathrm{H}_{7} \mathrm{~N}_{2} \mathrm{~F}_{5} \mathrm{O}_{2}$ : 258.0428, found 258.0423.

9-(Pentafluoroethyl)phenanthrene $(\mathbf{6 j})$ : Eluent: petroleum ether, $R_{\mathrm{f}}=0.7$. White solid (130 mg, 88\%), m.p. 54 $56{ }^{\circ} \mathrm{C} .{ }^{1} \mathrm{H}$ NMR $\left(400 \mathrm{MHz}, \mathrm{CDCl}_{3}, 293 \mathrm{~K}\right) \delta: 8.75(\mathrm{~d}, J=$ $8.2 \mathrm{~Hz}, 1 \mathrm{H}), 8.67$ (d, $J=8.4 \mathrm{~Hz}, 1 \mathrm{H}), 8.35$ (d, $J=7.6 \mathrm{~Hz}$, $1 \mathrm{H}), 8.18(\mathrm{~s}, 1 \mathrm{H}), 7.96(\mathrm{~d}, J=7.9 \mathrm{~Hz}, 1 \mathrm{H}), 7.70-7.73(\mathrm{~m}$, $4 \mathrm{H}) ;{ }^{19} \mathrm{~F}$ NMR $\left(375 \mathrm{MHz}, \mathrm{CDCl}_{3}\right) \delta$ : $-82.8(\mathrm{~s}, 3 \mathrm{~F})$, $-108.1(\mathrm{~s}, 2 \mathrm{~F}) ;{ }^{13} \mathrm{C} \mathrm{NMR}\left(101 \mathrm{MHz}, \mathrm{CDCl}_{3}, 293 \mathrm{~K}\right) \delta$ : $131.8,131.0,130.0,129.9,129.8,129.4,129.1,127.5$, $127.3,127.1,125.7 \sim 125.5(\mathrm{~m}), 123.2,122.6$; MS (EI) $m / z: 227,296$ (100). HRMS (EI) calcd for $\mathrm{C}_{16} \mathrm{H}_{9} \mathrm{~F}_{5}$ 296.0624, found 296.0622.

4-(Pentafluoroethyl)dibenzo[b,d]thiophene (6k): Eluent: petroleum ether, $R_{\mathrm{f}}=0.6$. Colorless liquid (148 mg, 98\%). ${ }^{1} \mathrm{H}$ NMR (400 MHz, $\left.\mathrm{CDCl}_{3}, 293 \mathrm{~K}\right) \delta: 8.29$ (d, $J=7.8 \mathrm{~Hz}$, $1 \mathrm{H}), 8.14(\mathrm{~d}, J=7.6 \mathrm{~Hz}, 1 \mathrm{H}), 7.86(\mathrm{~d}, J=7.2 \mathrm{~Hz}, 1 \mathrm{H}), 7.70$ $(\mathrm{d}, J=7.5 \mathrm{~Hz}, 1 \mathrm{H}), 7.58 \sim 7.44(\mathrm{~m}, 3 \mathrm{H}) ;{ }^{19} \mathrm{~F}$ NMR $(375$ $\left.\mathrm{MHz}, \mathrm{CDCl}_{3}\right) \delta:-84.3(\mathrm{~s}, 3 \mathrm{~F}),-113.9(\mathrm{~s}, 2 \mathrm{~F}) ;{ }^{13} \mathrm{C}$ NMR (101 MHz, $\left.\mathrm{CDCl}_{3}, 293 \mathrm{~K}\right) \delta: 139.5 \sim 139.3(\mathrm{~m})$, 137.6, 137.4, 133.9, 127.6, 126.1 (t, $J=6.9 \mathrm{~Hz}), 124.8$, $124.7,124.3,122.3,121.6$; IR (KBr) $v_{\max }$ : 3068, 2924, $1942,1819,1458,1446,1398,1336,1325,1280,1250$, $1209,1152,1135,1099,1084,1052,1023,979,938,910$, $854,813,798,751,730,714,707,658,630 \mathrm{~cm}^{-1}$; MS (EI) $m / z$ : 233 (100), 302. HRMS (EI) calcd for $\mathrm{C}_{14} \mathrm{H}_{7} \mathrm{~F}_{5} \mathrm{~S}$ 302.0189 , found 302.0186 .

3-(Pentafluoroethyl)-9-phenyl-9H-carbazole (61): ${ }^{[11]}$ El- 
uent: petroleum ether, $R_{\mathrm{f}}=0.5$. Colorless liquid (190 mg, 99\%). ${ }^{1} \mathrm{H}$ NMR (400 MHz, $\left.\mathrm{CDCl}_{3}, 293 \mathrm{~K}\right) \delta: 8.50(\mathrm{~s}, 1 \mathrm{H})$, $8.25(\mathrm{~d}, J=7.7 \mathrm{~Hz}, 1 \mathrm{H}), 7.68(\mathrm{t}, J=8.8 \mathrm{~Hz}, 3 \mathrm{H}), 7.60 \sim$ $7.57(\mathrm{~m}, 3 \mathrm{H}), 7.53 \sim 7.50(\mathrm{~m}, 2 \mathrm{H}), 7.47(\mathrm{t}, J=6.8 \mathrm{~Hz}, 1 \mathrm{H})$, $7.41(\mathrm{t}, J=6.8 \mathrm{~Hz}, 1 \mathrm{H}) ;{ }^{19} \mathrm{~F}$ NMR $\left(375 \mathrm{MHz}, \mathrm{CDCl}_{3}\right) \delta$ : $-84.7(\mathrm{~s}, 3 \mathrm{~F}),-112.5(\mathrm{~s}, 2 \mathrm{~F})$; IR (KBr) $v_{\max }: 3066,2923$, 1633, 1600, 1504, 1490, 1459, 1437, 1366, 1335, 1321, $1259,1238,1204,1146,1122,1110,1085,1028,1015$, 979, 940, 908, 893, 815, 803, 762, 747, 734, 699, 672, 641 $\mathrm{cm}^{-1}$; MS (EI) m/z: 292 (100), 361. HRMS (EI) calcd for $\mathrm{C}_{20} \mathrm{H}_{12} \mathrm{NF}_{5}$ 361.0890, found 361.0884.

4.6 General procedure for pentafluoroethylation of heteroarenes

Heteroarene (0.5 mmol), DBU (114 mg, $0.750 \mathrm{mmol}$, 1.50 equiv.) and $4 \mathbf{b}$ (302 $\mathrm{mg}, 0.750 \mathrm{mmol}, 1.50$ equiv.) were placed into an oven-dried Schlenk tube that was equipped with a stirring bar under $\mathrm{N}_{2} .10 .0 \mathrm{~mL}$ of freshly distilled DMSO was added and the tube was quickly sealed with a rubber stopper. The mixture was stirred under LED blue for $12 \mathrm{~h} .20 \mathrm{~mL}$ of water and $40 \mathrm{~mL}$ of ether were added to the mixture and the organic phase was separated and extracted with water $(10 \mathrm{~mL} \times 5)$, dried over anhydrous $\mathrm{Na}_{2} \mathrm{SO}_{4}$, and concentrated in vacuo. The residue was purified by flash chromatography on silica gel.

2-(Pentafluoroethyl)-1-phenyl-1 $H$-pyrrole (7a): Eluent: petroleum ether, $R_{\mathrm{f}}=0.7$. Colorless liquid (91 mg, 70\%); ${ }^{1} \mathrm{H}$ NMR $\left(400 \mathrm{MHz}, \mathrm{CDCl}_{3}, 293 \mathrm{~K}\right) \delta: 7.45 \sim 7.42(\mathrm{~m}$, $3 \mathrm{H}), 7.39 \sim 7.36(\mathrm{~m}, 2 \mathrm{H}), 6.90 \sim 6.89(\mathrm{~m}, 1 \mathrm{H}), 6.73(\mathrm{~s}$, $1 \mathrm{H}), 6.32 \sim 6.30(\mathrm{~m}, 1 \mathrm{H}) ;{ }^{19} \mathrm{~F}$ NMR $\left(375 \mathrm{MHz}, \mathrm{CDCl}_{3}\right) \delta$ : $-83.1(\mathrm{t}, 3 \mathrm{~F}),-102.7(\mathrm{~s}, 2 \mathrm{~F}) ;{ }^{13} \mathrm{C} \mathrm{NMR}(101 \mathrm{MHz}$, $\left.\mathrm{CDCl}_{3}, 293 \mathrm{~K}\right) \delta: 139.6,128.8,128.7,128.6,127.2,114.4$, 108.6; IR (KBr) $v_{\text {max }}: 3072,2927,1598,1543,1501,1461$, 1434, 1368, 1329, 1270, 1208, 1111, 1088, 1073, 1050, 1029, 1013, 1001, 926, 883, 813, 768, 745, 734, $697 \mathrm{~cm}^{-1}$; MS (EI) $m / z: 192$ (100), 261. HRMS (EI) calcd for $\mathrm{C}_{12} \mathrm{H}_{8} \mathrm{NF}_{5}: 261.0577$, found 261.0583.

1-(4-Chlorophenyl)-2-(pentafluoroethyl)- $1 H$-pyrrole (7b): Eluent: petroleum ether, $R_{\mathrm{f}}=0.7$. Yellow liquid (100 mg, 68\%); ${ }^{1} \mathrm{H}$ NMR (400 MHz, $\left.\mathrm{CDCl}_{3}, 293 \mathrm{~K}\right) \delta: 7.44 \sim$ $7.38(\mathrm{~m}, 2 \mathrm{H}), 7.31(\mathrm{~d}, J=8.7 \mathrm{~Hz}, 2 \mathrm{H}), 6.86 \sim 6.85(\mathrm{~m}$, $1 \mathrm{H}), 6.74 \sim 6.73(\mathrm{~m}, 1 \mathrm{H}), 6.34 \sim 6.30(\mathrm{~m}, 1 \mathrm{H}) ;{ }^{19} \mathrm{~F}$ NMR $\left(375 \mathrm{MHz}, \mathrm{CDCl}_{3}\right) \delta:-83.1(\mathrm{t}, J=2.7 \mathrm{~Hz}, 3 \mathrm{~F}),-102.8$ $(\mathrm{s}, 2 \mathrm{~F}) ;{ }^{13} \mathrm{C} \mathrm{NMR}\left(101 \mathrm{MHz}, \mathrm{CDCl}_{3}, 293 \mathrm{~K}\right) \delta: 138.0$, 134.6, 128.9, 128.5, 128.4, 114.7 114.5 (m), 109.0; IR $(\mathrm{KBr}) v_{\max }: 2956,2926,2855,1545,1497,1461,1435$, $1408,1368,1331,1270,1210,1130,1110,1093,1050$, 1039, 1019, 1011, 924, 883, 836, 749, 734, 716, $645 \mathrm{~cm}^{-1}$; MS (EI) $m / z: 226$ (100), 261, 295 (49.8), 297 (17.0). HRMS (EI) calcd for $\mathrm{C}_{12} \mathrm{H}_{7} \mathrm{NF}_{5} \mathrm{Cl}$ : 295.0187, found 295.0192.

1-(3-Bromophenyl)-2-(pentafluoroethyl)- $1 H$-pyrrole (7c): Eluent: petroleum ether, $R_{\mathrm{f}}=0.6$. Colorless liquid (122 mg, 72\%); ${ }^{1} \mathrm{H}$ NMR (400 MHz, $\left.\mathrm{CDCl}_{3}, 293 \mathrm{~K}\right) \delta$ : $7.58(\mathrm{dt}, J=6.9,1.9 \mathrm{~Hz}, 1 \mathrm{H}), 7.56(\mathrm{~s}, 1 \mathrm{H}), 7.37 \sim 7.28(\mathrm{~m}$, $2 \mathrm{H}), 6.87(\mathrm{dd}, J=2.5,1.3 \mathrm{~Hz}, 1 \mathrm{H}), 6.74(\mathrm{dd}, J=2.5,1.2$ $\mathrm{Hz}, 1 \mathrm{H}), 6.32(\mathrm{dd}, J=3.6,3.0 \mathrm{~Hz}, 1 \mathrm{H}) ;{ }^{19} \mathrm{~F} \mathrm{NMR}(375$
$\left.\mathrm{MHz}, \mathrm{CDCl}_{3}\right) \delta:-83.1(\mathrm{t}, J=2.7 \mathrm{~Hz}, 3 \mathrm{~F}),-102.7(\mathrm{~s}$, $2 \mathrm{~F}) ;{ }^{13} \mathrm{C} \mathrm{NMR}\left(101 \mathrm{MHz}, \mathrm{CDCl}_{3}, 293 \mathrm{~K}\right) \delta: 140.6,131.8$, $130.4,129.9,128.5,125.9,122.0,114.8 \sim 114.6(\mathrm{~m})$, 109.1; IR (KBr) $v_{\max }: 3131,1593,1578,1545,1486,1459$, 1438, 1366, 1329, 1272, 1209, 1113, 1090, 1051, 1041, 1018, 931, 882, 814, 787, 737, 690, $658 \mathrm{~cm}^{-1}$; MS (EI) $m / z: 45$ (100), 191, 226, 339 (18.1), 341 (15.6). HRMS (EI) calcd for $\mathrm{C}_{12} \mathrm{H}_{7} \mathrm{NF}_{5} \mathrm{Br} 338.9682$, found 338.9679 .

2-(Pentafluoroethyl)-1-(4-(trifluoromethyl)phenyl)-1 $H$ pyrrole (7d): Eluent: petroleum ether, $R_{\mathrm{f}}=0.7$. Colorless liquid (111 mg, 68\%); ${ }^{1} \mathrm{H}$ NMR (400 MHz, $\left.\mathrm{CDCl}_{3}, 293 \mathrm{~K}\right)$ $\delta: 7.72(\mathrm{~d}, J=8.2 \mathrm{~Hz}, 2 \mathrm{H}), 7.51(\mathrm{~d}, J=8.0 \mathrm{~Hz}, 2 \mathrm{H}), 6.90$ $(\mathrm{s}, 1 \mathrm{H}), 6.77(\mathrm{~s}, 1 \mathrm{H}), 6.36(\mathrm{t}, J=2.7 \mathrm{~Hz}, 1 \mathrm{H}) ;{ }^{19} \mathrm{~F}$ NMR $\left(375 \mathrm{MHz}, \mathrm{CDCl}_{3}\right) \delta:-62.7(\mathrm{~s}, 3 \mathrm{~F}),-83.2(\mathrm{t}, J=2.7$ $\mathrm{Hz}, 3 \mathrm{~F}),-102.5$ (s, 2F); ${ }^{13} \mathrm{C}$ NMR (101 $\mathrm{MHz}, \mathrm{CDCl}_{3}$, 293 K) $\delta: 143.5,131.8(\mathrm{q}, J=33.0 \mathrm{~Hz}), 129.4,128.5$, $127.0(\mathrm{q}, J=3.4 \mathrm{~Hz}), 124.6(\mathrm{q}, J=272.4 \mathrm{~Hz}), 116.3 \sim$ $115.9(\mathrm{~m}), 110.4$; IR $(\mathrm{KBr}) v_{\max }: 3137,1619,1547,1522$, $1463,1436,1418,1366,1327,1271,1212,1172,1134$, $1108,1090,1069,1050,1040,1020,1012,925,883,851$, $735,700 \mathrm{~cm}^{-1}$; MS (EI) m/z: 260 (100), 329. HRMS (EI) calcd for $\mathrm{C}_{13} \mathrm{H}_{7} \mathrm{NF}_{8}$ 329.0451, found 329.0452.

1-(4-Fluorophenyl)-2-(pentafluoroethyl)-1H-pyrrole (7e): Eluent: petroleum ether, $R_{\mathrm{f}}=0.6$. Colorless liquid (92 $\mathrm{mg}, 66 \%$ ); ${ }^{1} \mathrm{H}$ NMR (400 MHz, $\left.\mathrm{CDCl}_{3}, 293 \mathrm{~K}\right) \delta: 7.34$ (dd, $J=8.2,4.9 \mathrm{~Hz}, 2 \mathrm{H}), 7.11(\mathrm{t}, J=8.5 \mathrm{~Hz}, 2 \mathrm{H}), 6.86(\mathrm{~s}, 1 \mathrm{H})$, $6.72(\mathrm{~s}, 1 \mathrm{H}), 6.31(\mathrm{t}, J=2.8 \mathrm{~Hz}, 1 \mathrm{H}) ;{ }^{19} \mathrm{~F}$ NMR $(375 \mathrm{MHz}$, $\left.\mathrm{CDCl}_{3}\right) \delta:-83.2(\mathrm{t}, J=2.6 \mathrm{~Hz}, 3 \mathrm{~F}),-102.9(\mathrm{~s}, 2 \mathrm{~F})$ $-112.5 \sim-112.6(\mathrm{~m}, 1 \mathrm{~F}) ;{ }^{13} \mathrm{C}$ NMR $\left(101 \mathrm{MHz}, \mathrm{CDCl}_{3}\right.$, $293 \mathrm{~K}) \delta 163.7,161.2,135.6(\mathrm{~d}, J=2.9 \mathrm{~Hz}), 129.1(\mathrm{~d}, J=$ $8.8 \mathrm{~Hz}), 128.8,115.7(\mathrm{~d}, J=22.9 \mathrm{~Hz}), 114.6 \sim 114.4(\mathrm{~m})$, 108.8; IR (KBr) $v_{\max }: 3124,2924,1605,1545,1515,1464$, 1436, 1367, 1331, 1271, 1209, 1155, 1129, 1109, 1089, 1050, 1012, 926, 883, 843, 819, 746, $734 \mathrm{~cm}^{-1}$; MS (EI) $m / z$ : 210 (100), 279. HRMS (EI) calcd for $\mathrm{C}_{12} \mathrm{H}_{7} \mathrm{NF}_{6}$ : 279.0483 , found 279.0474 .

2-(4-Chlorophenyl)-3-(pentafluoroethyl)- $1 H$-indole (7f): Eluent: $V($ ethyl acetate $) / V($ petroleum ether $)=1 / 15, R_{\mathrm{f}}=$ 0.3. White solid (97 mg, 56\%), m.p. 99 101 ${ }^{\circ} \mathrm{C} ;{ }^{1} \mathrm{H}$ NMR $\left(400 \mathrm{MHz}, \mathrm{CDCl}_{3}, 293 \mathrm{~K}\right) \delta: 8.33(\mathrm{~s}, 1 \mathrm{H}), 7.76(\mathrm{~d}, J=7.8$ $\mathrm{Hz}, 1 \mathrm{H}), 7.45$ (q, $J=8.8 \mathrm{~Hz}, 4 \mathrm{H}), 7.41(\mathrm{~d}, J=8.5 \mathrm{~Hz}, 1 \mathrm{H})$, $7.31(\mathrm{t}, J=7.2 \mathrm{~Hz}, 1 \mathrm{H}), 7.25(\mathrm{t}, J=7.2 \mathrm{~Hz}, 1 \mathrm{H}) ;{ }^{19} \mathrm{~F}$ NMR $\left(375 \mathrm{MHz}, \mathrm{CDCl}_{3}\right) \delta:-84.6(\mathrm{t}, J=2.9 \mathrm{~Hz}, 3 \mathrm{~F}),-106.5$ $(\mathrm{s}, 2 \mathrm{~F}) ;{ }^{13} \mathrm{C}$ NMR $\left(101 \mathrm{MHz}, \mathrm{CDCl}_{3}, 293 \mathrm{~K}\right) \delta: 138.5(\mathrm{t}$, $J=4.6 \mathrm{~Hz}), 135.6,135.1,130.8,129.8,128.6,125.9$ (t, $J=$ $3.4 \mathrm{~Hz}), 123.7,121.8,120.6,111.0,101.7(\mathrm{t}, J=27.1 \mathrm{~Hz})$; IR (KBr) $v_{\max }: 3419,3065,2925,1621,1601,1572,1556$, $1520,1488,1456,1438,1400,1332,1315,1272,1201$, 1143, 1087, 1047, 1016, 932, 834, 763, 752, 725, 707, 652, $635 \mathrm{~cm}^{-1}$; MS (EI) m/z: 276 (100), 345 (69.4), 347 (23.5). HRMS (EI) calcd for $\mathrm{C}_{16} \mathrm{H}_{9} \mathrm{NF}_{5} \mathrm{Cl} 345.0344$, found 345.0338 .

2-(3-Chloro-4-fluorophenyl)-3-(pentafluoroethyl)- $1 \mathrm{H}$ indole (7g): Eluent: $V($ ethyl acetate $) / V$ (petroleum ether $)=$ $1 / 15, R_{\mathrm{f}}=0.3$. Yellow liquid $(109 \mathrm{mg}, 60 \%) ;{ }^{1} \mathrm{H} \mathrm{NMR}$ $\left(400 \mathrm{MHz}, \mathrm{CDCl}_{3}, 293 \mathrm{~K}\right) \delta: 8.38(\mathrm{~s}, 1 \mathrm{H}), 7.77$ (d, $J=7.8$ $\mathrm{Hz}, 1 \mathrm{H}), 7.60$ (dd, $J=6.6,1.3 \mathrm{~Hz}, 1 \mathrm{H}), 7.42$ (d, $J=8.0 \mathrm{~Hz}$, 
2H), $7.33(\mathrm{t}, J=7.2 \mathrm{~Hz}, 1 \mathrm{H}), 7.21 \sim 7.30(\mathrm{~m}, 2 \mathrm{H}) ;{ }^{19} \mathrm{~F}$ NMR (375 MHz, $\left.\mathrm{CDCl}_{3}\right) \delta$ : $-84.7(\mathrm{t}, J=3.1 \mathrm{~Hz}, 3 \mathrm{~F})$, $-106.6(\mathrm{~s}, 2 \mathrm{~F}),-113.6(\mathrm{ddd}, J=8.4,7.0,4.6 \mathrm{~Hz}, 1 \mathrm{~F})$; ${ }^{13} \mathrm{C}$ NMR (101 MHz, $\left.\mathrm{CDCl}_{3}, 293 \mathrm{~K}\right) \delta: 160.0,157.4,137.1$ (t, $J=4.4 \mathrm{~Hz}), 135.1,131.8,129.6$ (d, $J=7.2 \mathrm{~Hz}), 128.4$ (d, $J=4.1 \mathrm{~Hz}), 125.8$ (t, $J=3.0 \mathrm{~Hz}), 123.9,122.0,120.6$, $116.6(\mathrm{~d}, J=21.5 \mathrm{~Hz}), 111.1,102.1(\mathrm{t}, J=27.2 \mathrm{~Hz})$; IR $(\mathrm{KBr}) v_{\max }: 3458,3398,3062,1622,1560,1493,1458$, 1439, 1389, 1368, 1330, 1315, 1264, 1248, 1204, 1147, 1131, 1099, 1065, 1049, 1016, 942, 921, 890, 868, 827, 806, 763, 750, 728, 714, $694 \mathrm{~cm}^{-1}$; MS (EI) m/z: 294 (100), 363 (56.7), 365 (20.1). HRMS (EI) calcd for $\mathrm{C}_{16} \mathrm{H}_{8} \mathrm{NF}_{6} \mathrm{Cl} 363.0249$, found 363.0247.

2-(Naphthalen-2-yl)-3-(pentafluoroethyl)- $H$-indole (7h): Eluent: $V$ (ethyl acetate $) / V($ petroleum ether $)=1 / 15, R_{\mathrm{f}}$ $=0.2$. Yellow liquid $(121 \mathrm{mg}, 67 \%) ;{ }^{1} \mathrm{H}$ NMR $(400 \mathrm{MHz}$, $\left.\mathrm{CDCl}_{3}, 293 \mathrm{~K}\right) \delta: 8.45(\mathrm{~s}, 1 \mathrm{H}), 8.03(\mathrm{~s}, 1 \mathrm{H}), 7.95 \sim 7.89$ $(\mathrm{m}, 3 \mathrm{H}), 7.81(\mathrm{~d}, J=7.8 \mathrm{~Hz}, 1 \mathrm{H}), 7.64(\mathrm{~d}, J=8.3 \mathrm{~Hz}, 1 \mathrm{H})$, $7.62 \sim 7.53(\mathrm{~m}, 2 \mathrm{H}), 7.44(\mathrm{~d}, J=7.9 \mathrm{~Hz}, 1 \mathrm{H}), 7.33(\mathrm{t}, J=$ $7.1 \mathrm{~Hz}, 1 \mathrm{H}), 7.29(\mathrm{~d}, J=8.0 \mathrm{~Hz}, 1 \mathrm{H}) ;{ }^{19} \mathrm{~F}$ NMR $(375 \mathrm{MHz}$, $\left.\mathrm{CDCl}_{3}\right) \delta:-89.8(\mathrm{t}, J=3.2 \mathrm{~Hz}, 3 \mathrm{~F}),-111.6(\mathrm{~s}, 2 \mathrm{~F}) ;{ }^{13} \mathrm{C}$ NMR (101 MHz, $\left.\mathrm{CDCl}_{3}, 293 \mathrm{~K}\right) \delta: 139.9$ (t, $\left.J=4.4 \mathrm{~Hz}\right)$, $135.2,133.3,132.7,129.1,128.8,128.3,128.0,127.8$, $127.1,126.8,126.7,126.1$ (t, $J=3.3 \mathrm{~Hz}), 123.5,121.7$, $120.6,111.0,101.5$ (t, $J=27.2 \mathrm{~Hz})$; IR $(\mathrm{KBr}) v_{\max }: 3405$, 3059, 2925, 2854, 1558, 1507, 1470, 1456, 1439, 1430, 1329, 1268, 1204, 1157, 1141, 1128, 1088, 1047, 1017, 960, 931, 900, 861, 822, 793, 748, $711 \mathrm{~cm}^{-1}$; MS (EI) $\mathrm{m} / \mathrm{z}$ : 361 (100). HRMS (EI) calcd for $\mathrm{C}_{20} \mathrm{H}_{12} \mathrm{NF}_{5}$ 361.0890, found 361.0887 .

3-(Pentafluoroethyl)-2-phenyl-1H-indole (7i): Eluent: $V($ ethyl acetate $) / V($ petroleum ether $)=1 / 15, R_{\mathrm{f}}=0.4$. White solid (95 mg, 61\%), m.p. 97 99 ${ }^{\circ} \mathrm{C} ;{ }^{1} \mathrm{H}$ NMR (400 MHz, $\left.\mathrm{CDCl}_{3}, 293 \mathrm{~K}\right) \delta: 8.34(\mathrm{~s}, 1 \mathrm{H}), 7.77(\mathrm{~d}, J=7.9 \mathrm{~Hz}, 1 \mathrm{H})$, $7.56 \sim 7.52(\mathrm{~m}, 2 \mathrm{H}), 7.50 \sim 7.45(\mathrm{~m}, 2 \mathrm{H}), 7.42 \sim 7.39(\mathrm{~m}$, $1 \mathrm{H}), 7.40(\mathrm{~d}, J=7.8 \mathrm{~Hz}, 1 \mathrm{H}), 7.33 \sim 7.27(\mathrm{~m}, 1 \mathrm{H}), 7.27 \sim$ $7.22(\mathrm{~m}, 1 \mathrm{H}) ;{ }^{19} \mathrm{~F}$ NMR $\left(375 \mathrm{MHz}, \mathrm{CDCl}_{3}\right) \delta$ : $-84.6(\mathrm{t}$, $J=3.1 \mathrm{~Hz}, 3 \mathrm{~F}),-106.5(\mathrm{~s}, 2 \mathrm{~F}) ;{ }^{13} \mathrm{C} \mathrm{NMR}(101 \mathrm{MHz}$, $\left.\mathrm{CDCl}_{3}, 293 \mathrm{~K}\right) \delta: 139.9(\mathrm{t}, J=4.4 \mathrm{~Hz}), 135.2,133.3,132.7$, $129.1,128.8,128.3,128.0,127.8,127.1,126.8,126.7$, $126.1(\mathrm{t}, J=3.3 \mathrm{~Hz}), 123.5,121.7,120.6,111.0,101.5(\mathrm{t}$, $J=27.2 \mathrm{~Hz}$ ); IR (KBr) $v_{\max }: 3404,3061,2924,1622,1559$, $1492,1458,1449,1433,1369,1330,1268,1201,1160$, 1143, 1119, 1093, 1049, 1028, 1001, 934, 770, 761, 749, $699 \mathrm{~cm}^{-1}$; MS (EI) m/z: 242 (100), 311. HRMS (EI) calcd for $\mathrm{C}_{16} \mathrm{H}_{10} \mathrm{NF}_{5} 311.0733$, found 311.0735 .

1-Methyl-3-(pentafluoroethyl)-2-phenyl-1H-indole (7j):
Eluent: petroleum ether $\left(R_{\mathrm{f}}=0.3\right)$. White solid $(93 \mathrm{mg}$, 57\%), m.p. 77 $79{ }^{\circ} \mathrm{C} ;{ }^{1} \mathrm{H}$ NMR (400 MHz, $\mathrm{CDCl}_{3}, 293$ K) $\delta: 7.76(\mathrm{~d}, J=7.6 \mathrm{~Hz}, 1 \mathrm{H}), 7.48 \sim 7.46(\mathrm{~m}, 3 \mathrm{H}), 7.40 \sim$ $7.32(\mathrm{~m}, 4 \mathrm{H}), 7.27 \sim 7.24(\mathrm{~m}, 1 \mathrm{H}), 3.49(\mathrm{~s}, 3 \mathrm{H}) ;{ }^{19} \mathrm{~F}$ NMR $\left(375 \mathrm{MHz}, \mathrm{CDCl}_{3}\right) \delta$ : $-84.9(\mathrm{t}, J=3.3 \mathrm{~Hz}, 3 \mathrm{~F}),-106.5$ (s, 2F); IR (KBr) $v_{\max }: 3058,2982,2948,1608,1579$, $1555,1495,1484,1470,1445,1435,1404,1377,1358$, $1327,1278,1239,1217,1197,1175,1155,1117,1092$, 1075, 1056, 1031, 1003, 925, 918, 843, 800, 762, 750, 736, $702 \mathrm{~cm}^{-1}$; MS (EI) m/z: 256 (100), 325. HRMS (EI) calcd for $\mathrm{C}_{17} \mathrm{H}_{12} \mathrm{NF}_{5}$ 325.0890, found 325.0883.

Supporting information ${ }^{1} \mathrm{H}$ NMR, ${ }^{19} \mathrm{~F}$ NMRand ${ }^{13} \mathrm{C}$ NMR spectra of compounds $4 \mathbf{a} / \mathbf{4} \mathbf{b}, \mathbf{5 a} \sim \mathbf{5 i}, \mathbf{6 a} \sim \mathbf{6} \mathbf{l}$ and $\mathbf{7} \mathbf{a} \sim \mathbf{7 j}$. The Supporting Information is available free of charge via the Internet at http://sioc-journal.cn/.

\section{References}

[1] Liu, Y.-F.; Shao, X.-X.; Zhang, P.-P.; Shen, Q. Org. Lett. 2015, 17, 2752.

[2] (a) Trost, B. M.; Melvin, L. S. Sulfur Ylide: Emerging Synthetic Intermediates, Academic Press, New York, 1975.

(b) Aggrawal, V. K.; Winn, C. L. Acc. Chem. Res. 2004, 37, 611.

(c) Tang, Y.; Ye, S.; Sun, X-L. Synlett 2005, 18, 2720.

(d) Burtoloso, A. C. B.; Dias, R. M. P.; Leonarczyk, I. A. Eur.-J. Org. Chem. 2013, 5005.

[3] Zhu, J.-S.; Liu, Y.-F.; Shen, Q. Angew. Chem., Int. Ed. 2016, 55, 9050.

[4] Liu, Y.-F.; Lu, L.; Shen, Q. Angew. Chem., Int. Ed. 2017, 56, 9930.

[5] (a) Yagupolskii, L. M.; Mironova, I. I.; Maletina, I. I.; Orda, V. V. Synthesis 1978, 835.

(b) Yagupolskii, L. M. J. Fluorine Chem. 1987, 36, 1.

(c) Umemoto, T.; Kuriu, Y.; Shuyama, H.; Miyano, O.; Nakayama, S. I. J. Fluorine Chem. 1982, 80, 695

(d) Umemoto, T.; Kuriu, Y.; Shuyama, H.; Miyano, O.; Nakayama, S. I. J. Fluorine Chem. 1986, 31, 37.

(f) Zhdankin, V. V.; Kuehl, C. J. Tetrahedron Lett. 1994, 35, 1809. (g) Umemoto, T.; Kuriu, T. Tetrahedron Lett. 1981, 22, 5197. (h) Umemoto, T.; Kuriu, T. Chem. Lett. 1982, 65.

[6] Liu, Q.-H.; Xu, X.-H.; Qing, F.-L. J. Org. Chem. 2014, 79, 10434.

[7] Zhu, J.-S.; Li, Y.-G.; Ni, C.-F.; Shen, Q. Chin. J. Chem. 2016, 34, 662.

[8] Boiko, V. N.; Shchupak, G. M. J. Fluorine Chem. 1994, 3, 207.

[9] Zhang, C.-P.; Wang, Z.-L.; Chen, Q.-Y.; Zhang, C.-T.; Gu, Y.-C.; Xiao, J.-C. Angew. Chem., Int. Ed. 2011, 50, 1896.

[10] (a) Cheng, Y.-Z.; Yu, S.-Y. Org. Lett. 2016, 18, 2962. (b) Cheng, Y.-Z.; Yuan, X.-G.; Ma, J.; Yu. S.-Y. Chem.-Eur. J. 2015, 21,8355 .

[11] Huang, Y.-J.; Ajitha, M. J.; Huang, K.-W.; Zhang, Z.-X.; Weng, Z.-Q. Dalton Trans. 2016, 45, 8468. 